\title{
The Right Device for the Right Patient at the Right Time
}

\author{
Satoshi Yasukochi, MD
}

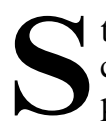
tent implantation for vascular or conduit stenosis of congenital heart disease (CHD) has been a common pediatric interventions in Japan for a long period. Stent therapy is performed in various clinical settings and is especially effective in cases of suboptimal or ineffective dilatation by balloon angioplasty. It also contributes greatly to improving the outcome of surgical results for CHD by rescuing lesions that are difficult to access or to manage by conventional surgery., 1,2

In this issue of the Journal, Tomita et $\mathrm{al}^{3}$ describe well and report on the current status of stent therapy for CHD in Japan. They analyzed web-based registry data between 2016 and 2018 of Japanese Society of Congenital Interventional Cardiology (JCIC; formerly the Japanese Society of Pediatric Interventional Cardiology (JPIC)), which covered $>90 \%$ of domestic institutions performing pediatric

\section{Article p 1517}

interventions. This means the nationwide survey of the JCIC registry provides a real-world data of stent therapy for CHD in Japan.

The notable points of Tomita et al's report are: (1) first nationwide analysis using registry data in this field, (2) analysis of the current status and effectiveness of stent therapy for each lesion with the type of stent use, and (3) analysis of the risk and adverse events at each age and the disparity between institutions.

During their 3-year study period, there were 470 procedures, 443 sessions in 391 cases. The ratio of the number of stent implantations to the total pediatric interventions ranged from $3.0 \%$ to $3.5 \%$ regardless of the increase in the

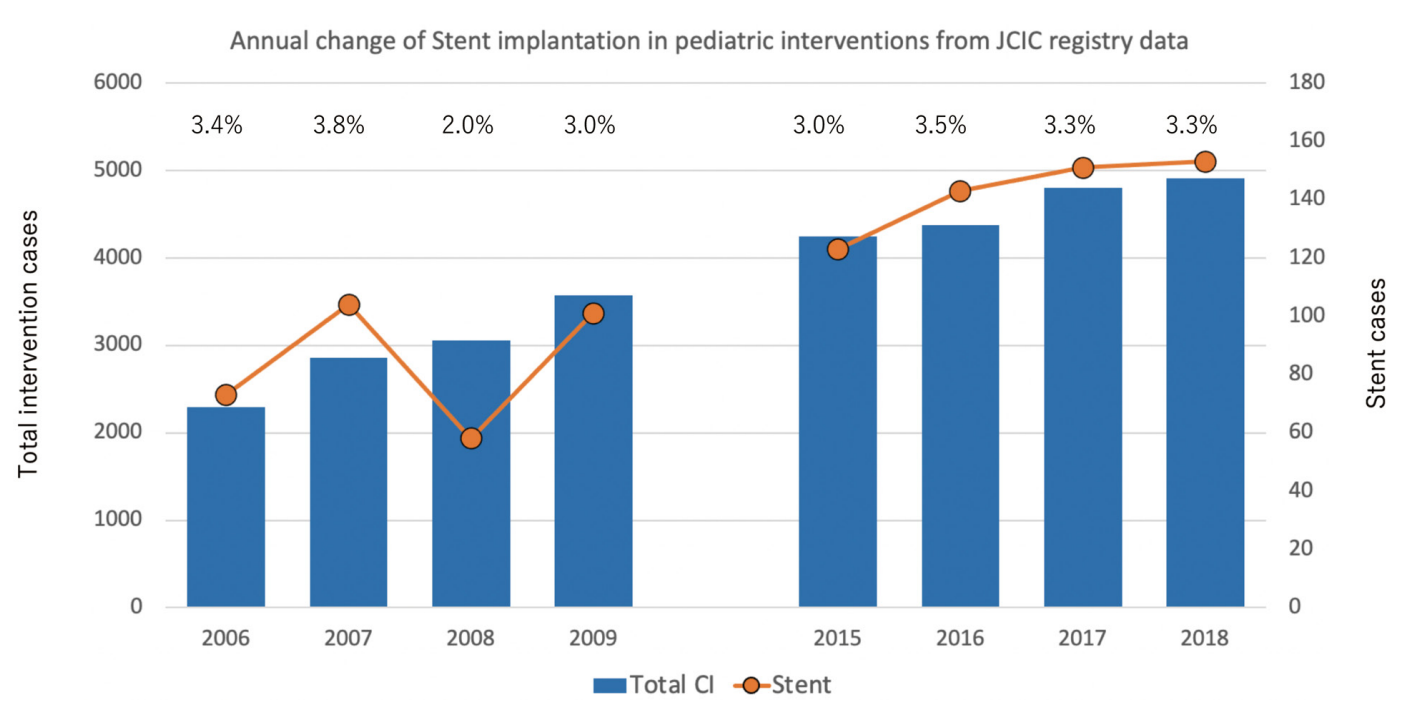

Figure. Data obtained from 2006 to 2015 by questionnaire survey of Japanese Society of Pediatric Interventional Cardiology (JPIC) for 14 leading institutions performing pediatric interventions (PI), and from 2016 to 2018 by web-based Japanese Society of Congenital Interventional Cardiology (formerly JPIC) registry for $>90 \%$ of total institutions performing PI in Japan. The line graph shows the number of stent implantations and the ratio of stent implantation to total $\mathrm{Cl}$ is indicated in the upper part of the graph as percentage.

The opinions expressed in this article are not necessarily those of the editors or of the Japanese Circulation Society.

Received April 12, 2021; accepted April 12, 2021; J-STAGE Advance Publication released online May 22, 2021

Heart Center, Nagano Children's Hospital, Azumino, Japan

Mailing address: Satoshi Yasukochi, MD, Director, Heart Center, Nagano Children's Hospital, 3100 Toyoshina, Azumino 399-8288,

Japan. E-mail: maplesyjuha@me.com

All rights are reserved to the Japanese Circulation Society. For permissions, please e-mail: cj@j-circ.or.jp

ISSN-1346-9843 
total number of procedures, which did not significantly change, compared with the $2.0-3.8 \%$ in the previous JPIC survey between 2006 and 2009 (Figure). ${ }^{46}$

The procedural success rate was reported as $96.4 \%$ among all ages, which is quite high. The complication rate was $12.4 \%$ and 4 deaths directly related to stent implantation $(1 \%)$ were reported; however, these hospital deaths and severe complications were found in the high-risk group with preoperative risk factors, including younger age $(<1$ year) and lower body weight at the time of stent implantation, and were not related to the institution's experience. According to the annual report of the JCIC, stent therapy has a higher complication rate than other pediatric interventional procedures at $3.8-4.1 \%$, which may be explained by characteristics of the stents themselves and related technical difficulties. ${ }^{\mathbf{4}} \mathbf{6}$

Recently, several reports using registry data were published for quality control and risk management of catheter interventions with the aim of safer and more efficient catheter treatments. The National Cardiovascular Data Registry Improving Pediatric and Adult Congenital Treatment (NCDR IMPACT) registry is a representative example. A proposed validated risk-standardization model for adverse outcomes after CHD catheterization can support reporting of risk-adjusted outcomes from the IMPACT registry for quality improvement. ${ }^{7,8}$ The authors created a risk adjustment model that included procedure-type risk category, a number of hemodynamic vulnerability indicators, renal insufficiency, single-ventricle physiology, and coagulation disorder from their derivation and validation cohort. The risk of major adverse events (MAE) and death from the IMPACT registry was reported as $7.1 \%$ and $2 \%$, respectively. They also reported the high-risk group for MAE was neonates $(22.2 \%)$, compared with adults $(5.3 \%)$, as Tomita et al report in this issue of the Journal.

The more important issue from the current report is the "off-label" use of stents in Japan, despite the fact that stent therapy is so widely performed in daily practice. The definition of "off-label" device use is that the treatment should be done under a doctor's license with sufficient informed consent from the patient and family. If something goes wrong with stent implantation and its follow-up, it cannot be legally warranted by the medical insurance system. This is a crucial issue for healthcare professionals and patients' families involved in this treatment. The issue of "off-label" device use is also an issue in the USA where many medical pediatric devices are often used "off-label" under a close relationship between patients and doctors, as in Japan. However, the situation around stent devices is somewhat better in the USA than in Japan, because they can use newgeneration stents under regulatory systems such as Humanitarian Device Exemption. ${ }^{9}$

Moreover, there is also the issue of "device lag" in Japan. We use a 1st-generation stent that is not used in other coun- tries. Recently, with the cooperation of government agencies and regulatory bodies, industries involved in device development, and academia, Harmonization-By-Doing for Children activities launched in 2016 have started to resolve this device-lag issue to get safer and faster approval of new devices, including stents, by utilizing the registry data. ${ }^{\mathbf{1 0}}$

Not only medical personnel but also pediatric patients are looking forward to having stents that can be implanted safely and more effectively, and expanded at the proper time as the patient grows to adult size.

In this context, we must take action to ensure "the right device for the right patient at the right time".

\section{References}

1. JCS/JCC/JSCS/JSVS/JATS 2021 Guideline on catheter intervention for congenital heart disease and structural heart disease 2021. https://www.j-circ.or.jp/guideline/guideline-series/ (accessed April 1, 2021).

2. Guideline of indication of catheter intervention for congenital heart disease and pediatric onset heart disease. The Japanese Society of Pediatric Cardiology and Cardiac Surgery-The Japanese Society of Pediatric Interventional Cardiology guideline committee. J Pediatr Cardiol Card Surg 2012; 28(Suppl): s1-s40.

3. Tomita H, Kim SH, Inuzuka R, Matsui H, Tachimori H, Kobayashi T, et al. Stent implantation for congenital heart disease in Japan: Comprehensive analysis from the Japanese Society of Congenital Interventional Cardiology Registry. Circ J 2021; 85: $1517-1524$.

4. Japanese Society of Congenital Interventional Cardiology. Result page of the annual surveillance. https://center6.umin.ac. jp/oasis/jpic/member/data.htm (accessed March 15, 2018).

5. Kim SH, Matsui H, Inuzaka R, Yoshimoto J, Aoki T, Kato A, et al. Nationwide survey of catheter intervention and ablation for congenital heart disease, Kawasaki disease, and tachyarrhythmias in 2018: Annual report of JCIC-registry. J Jpn Soc Congenital Interv Cardiol 2019; 4: 24-38.

6. Tomita H, Nakanishi T, Hamaoka K, Kobayashi T, Ono Y. Stenting in congenital heart disease: Medium- and long-term outcomes from the JPIC stent survey. Circ J 2010; 74: 16761683 .

7. Jayaram N, Spertus JA, Kennedy KF, Vincent R, Martin GR, Curtis JP, et al. Modeling major adverse outcomes of pediatric and adult patients with congenital heart disease undergoing cardiac catheterization: Observations from the NCDR IMPACT registry (National Cardiovascular Data Registry Improving Pediatric and Adult Congenital Treatment). Circulation 2017; 136: 20092019.

8. Stefanescu Schmidt AC, Armstrong A, Kennedy KF, Nykanen D, Aboulhosn J , Bhatt AB. Prediction of adverse events after catheter-based procedures in adolescents and adults with congenital heart disease in the IMPACT registry. Eur Heart $J$ 2017; 38: $2070-2076$.

9. Moscucci M, Carrol JD, Webb JG. General overview of interventions for structural heart disease. In: Moscucci M, editor. Grossman \& Baim's cardiac catheterization, angiography, and intervention, 8th edn. Wolters Kluwer/Lippincott Williams \& Wilkins, 2014: 760-771.

10. Takahashi S, Ibrahim N, Yasukochi S, Ringel R, Ing F, Tomita $\mathrm{H}$, et al; Harmonization by Doing for Children Working Group. Partnership between Japan and the United States for early development of pediatric medical devices: Harmonization By Doing for Children. Circ J 2020; 84: 786-791. 